\author{
Yunfei Xing \\ State Key Laboratory of High \\ Temperature Gas Dynamics (LHD), \\ Institute of Mechanics, \\ Chinese Academy of Sciences, \\ 100190 Beijing, China \\ e-mail: xingyunfei@imech.ac.cn
Institut für Thermodynamik der
Luft-und Raumfahrt (ITLR), Universität Stuttgart, Pfaffenwaldring 31 , 70569 Stuttgart, Germany \\ Bernhard Weigand
}

\section{Optimum Jet-to-Plate Spacing of Inline Impingement Heat Transfer for Different Crossflow Schemes}

\begin{abstract}
A nine-by-nine jet array impinging on a flat plate at Reynolds numbers from 15,000 to 35,000 has been studied by the transient liquid crystal method. The spacing between the impingement plate and target plate is adjusted to be 1,2,3,4, and 5 jet diameters. The effect of jet-to-plate spacing has been investigated for three jet-induced crossflow schemes, referred as minimum, medium, and maximum crossflow, correspondingly. The local air jet temperature is measured at several positions on the impingement plate to account for an appropriate reference temperature of the heat transfer coefficient. The jetto-plate spacing, $H / d=3$, is found to be better than the others for all the crossflow schemes. Jet-to-plate spacings $H / d=1$ and $H / d=2$ result in a sudden decrease in the stagnation zone. The large jet-to-plate spacings $H / d=4$ and $H / d=5$ could not provide higher heat transfer performance with higher crossflow. [DOI: 10.1115/1.4023562]
\end{abstract}

Keywords: impingement cooling, heat transfer, jet-to-plate spacing, crossflow

\section{Introduction}

The last 20 years have seen a large improvement in gas turbine technology, mainly due to an increase in turbine pressure ratios and turbine inlet temperature. The effect of firing temperature is very important, as for approximately every $55 \mathrm{~K}$ increase in temperature, the work output increases by approximately $10 \%$ and the overall efficiency by $1 \%-1.5 \%$, as shown by Boyce [1]. In practice, high-turbine inlet temperatures have been achieved because of the growth of material technology, new coatings, and new cooling schemes. Film cooling has become an established technology for the conventional cooling of turbine vanes and blades or combustor liners. In particular, for combustors, emission restrictions in recent years have accelerated the development of modern concepts, such as the dry low emission (DLE) combustor with low production of $\mathrm{NO}$ and $\mathrm{NO}_{2}(\mathrm{NOx})$ emissions. However, control of the NOx emissions requires a minimization of film cooling and dilution air. Consequently, these combustors are typically cooled by enhanced backside convective heat transfer. Combustion liners typically have a double-wall structure, and impingement cooling is often used to keep the cooling effectiveness high. Therefore, the impingement hole shape, size, and location have to be tailored to attain both sufficiently high average heat transfer coefficient and uniformity in the surface heat transfer distribution to avoid local hot or cold spots.

Hollworth and Berry [2] measured the heat transfer values for impinging jet arrays with large jet-to-jet spacings, which are $X / d=10,15,20$, and 25 . They found that the local heat transfer coefficients are influenced by interference from neighboring jets and the averaged heat transfer coefficient is sensitive to jet-toplate spacing only when the jet-to-jet spacing is less than five jet diameters. Metzger et al. [3] compared heat transfer characteristics for inline and staggered impingement patterns. The particular impingement geometries are in the range of $X / d=5$ and 10 ; $\mathrm{Y} / \mathrm{d}=4,6$, and 8 ; and $H / d=1-3$. They showed that the areaaveraged heat transfer coefficients for the inline pattern are, in

Contributed by the Heat Transfer Division of ASME for publication in the Journal OF Heat TRANSFER. Manuscript received September 26, 2011; final manuscript received January 9, 2013; published online June 17, 2013. Assoc. Editor: Phillip M. Ligrani. every case, equal to or larger than those for the corresponding staggered pattern. Although the spanwise heat transfer coefficients can vary significantly with $H / d$, especially with dense spanwise spacings, the area-averaged values remain relatively insensitive to jet-to-plate spacing. Andrews et al. [4] studied the effect of jet-tojet spacing for the impingement heat transfer by varying the diameter of the jet at a constant pitch $(\mathrm{X})$ and constant impingement gap $(\mathrm{H})$. There are six different jet diameters, corresponding to six jet-to-jet spacings (1.9-21.5) and jet-to-plate spacings (0.4-5.15). They concluded that there is little influence of $H / d$ on the heat transfer values. Obot and Trabold [5] made a comparison of the effects of three jet-induced crossflow schemes on impingement heat transfer. The study covered the jet Reynolds numbers of 1000 to 21,000 , jet-to-jet spacings $(X / d, Y / d)$ are $(10,8),(10,4)$, and $(5.6,4)$, and jet-to-plate spacings ranging from 2 to 12 . The results showed that the best heat transfer performance is obtained with minimum crossflow scheme. And there is no advantage in using dense spanwise spacing when designing for larger jet-toplate spacing. Van Treuren et al. [6] measured the local heat transfer coefficients of inline and staggered impinging jets array for the Reynolds numbers ranging from 10,000 to 40,000 and jet-to-plate spacings of 1,2 , and 4 . They found that the averaged Nusselt number value for $H / d=2$ is the highest with the value for $H / d=4$ being the lowest. San and Lai [7] investigated the influence of the jet-to-jet spacing on the staggered impingement heat transfer performance. The comparisons are based on the stagnation values. Jet spacing values $(X / d, Y / d)$ of $4,6,8,12$, and 16 , jet-to-plate spacings $(H / d)$ varying from 2 to 5 , and Reynolds numbers ranging from 10,000 to 30,000 have been investigated. They concluded that the optimum jet-to-jet spacing for the $H / d$ of 2 is 8 ; the optimum jet-to-jet spacing for the $H / d$ of 3 is 12 ; and the optimum jet-to-jet spacing for the $H / d$ of 5 is 6 . Garimella and Schroeder [8] investigated the local heat transfer distributions in confined multiple air jets for the jet-to-jet spacings of 3 and 4 and jet-to-plate spacings (0.5-4) and different Reynolds numbers (5000-20,000). The results showed that the averaged heat transfer coefficients are appreciably larger at lower jet-to-plate spacing; however, the heat transfer distribution over the stagnation point is more uneven at smaller $H / d$. Brevet et al. [9] studied the heat transfer characteristics of one row of impinging jets by varying 
the $Y / d$ from 2 to 10 with the same jet-to-jet spacing $X / d=10$. The jet-to-plate spacings $H / d=1,2,5$, and 10 , and Reynolds numbers ranging from 3000 to 20,000 were measured. They found that the optimum jet-to-plate spacing is within the range from 2 to 5. Bailey and Bunker [10] tested the local heat transfer coefficients for impinging jet arrays with the jet-to-jet spacings $X / d$ and $Y / d$ of 3, 6, and 9. The jet-to-plate spacings have been varied from 1.25 to 5.5 and Reynolds numbers ranging from 14,000 to 65,000 . They found that the heat transfer value for $H / d=2.75$ is better than those of $H / d=1.25$. While they did not compare the heat transfer values for different $H / d$ in detail, Gao [11] studied the effect of the jet-to-plate spacing on impingement heat transfer for $X / d=Y / d=4 ; H / d=1,3$, and 5; and Reynolds numbers ranging from 5000 to 15,000 . He found that the jet-to-plate spacing $H / d$ is optimum at a value of 3. Dano et al. [12] studied the effect of nozzle geometry on the heat transfer performances of a semiconfined impinging jets array. The results showed that the averaged Nusselt numbers decrease with increasing jet-to-plate spacing when $1 \leq H / d \leq 4$. Katti and Prabhu [13] investigated the influence of the spanwise jet-to-jet spacing on local heat transfer distribution for inline impingement arrays, the jet-to-plate spacings $(H / d=1$, 2, and 3), and Reynolds numbers ranging from 3000 to 10,000 . They also concluded that the averaged Nusselt numbers decrease with increasing jet-to-plate spacing. Geers et al. [14] reported the heat transfer performance of inline impinging jets arrays for the Reynolds numbers ranging from 5000 to 20,000 and jet-to-plate spacing $H / d=3,4,6,8$, and 10 . The experiments indicated that the multiple jet heat transfer is strongly influenced by jet interactions and the averaged Nusselt numbers decrease with increasing jet-to-plate spacing. Son et al. [15] performed a study of the impinging cooling system for different jet-to-jet spacings and jetto-plate spacing $H / d$ from 1.875 to 3 . They found that the change of jet diameter and crossflow can influence the heat transfer distribution on the target plate. Park et al. [16] investigated the influence of Mach number and Reynolds number on the impingement heat transfer for $H / d=3$ and Reynolds number ranging from 15,000 to 60,000 .

Figure 1 shows the dependency of the area-averaged Nusselt number on the jet-to-plate spacing for inline multiple jet configurations of different jet-to-jet spacings $(X / d, Y / d)$. One can see that the slopes of the changes in area-averaged Nusselt number with $H / d$ are significantly affected by the jet-to-jet spacing. The averaged heat transfer coefficient is more sensitive to jet-to-plate spacing for denser jet-to-jet spacing. Table 1 summarizes the relevant

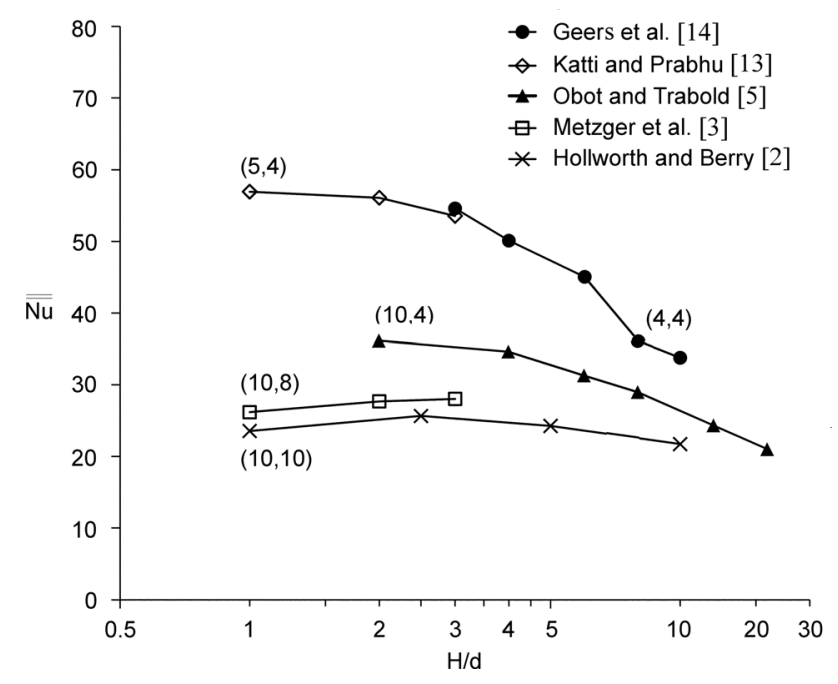

Fig. 1 Effect of jet-to-plate spacing on area-averaged Nusselt number for inline arrays with different jet-to-jet spacings at a Reynolds number of around 10,000 (numbers in brackets indicate jet-to-jet spacings $(X / d, Y / d))$ literature based on the particular impingement geometries for inline impingement. It may be noted that there are very detailed heat transfer results for the jet-to-jet spacing $X / d$ of round 10 . The jet-to-plate spacings covered from 1 to 10 for Reynolds numbers up to 65,050 , while for the dense jet-to-jet spacings $(X / d \leq 5)$, it appears that there is only little information available on the jet-toplate spacing $H / d$ of 1 . Only Metzger et al. [3] measured the heat transfer value for jet-to-plate spacing $H / d=1$ for the maximum crossflow scheme and the Reynolds number of 10,000 . Higher Reynolds numbers and local heat transfer values should be investigated. It has been consistently reported that the optimum jet-to-plate spacing for multijet impingement occurs between 1 and 3. Katti and Prabhu [13] found that $H / d=1$ reaches the highest heat transfer rate, while Geers et al. [14] reported that $H / d=3$ has the highest heat transfer rate. For dense jet-to-plate spacings, there is no information so far covering the jet-to-plate spacing from 1 to 5 .

In the present study, a large scale impingement array is used to accurately investigate the heat transfer for different impingement cooling configurations. Particular attention is paid to measure the effect of jet-to-plate spacing for different crossflow schemes. Results are evaluated in the form of local heat transfer coefficients, which provide significantly more detailed information than average values using the liquid crystal measurement method. The most important of the unexplored areas are the higher jet Reynolds numbers (Reynolds number from 15,000 to 35,000 ), three crossflow schemes (that is minimum crossflow, medium crossflow, and maximum crossflow), and the full coverage range at smaller jet-to-plate spacing $H / d$ from 1 to 5 . The present study will focus on the optimum jet-to-plate spacing for different crossflows.

\section{Experimental Setup}

2.1 Test Section. Figure 2 shows a sketch of the experimental setup. A vacuum pump system is used to generate the desired air flow in the test channel. The air enters the channel under atmospheric conditions via a filter and a heater. The heater consists of several meshes made out of stainless steel and is able to heat the air from ambient temperature up to $100^{\circ} \mathrm{C}$ in less than $0.3 \mathrm{~s}$. Downstream of the heater, the air enters the inlet plenum and then the impingement model shown in Fig. 3. This model is equipped with thermocouples and pressure taps for the measurement of the heat transfer and pressure loss. It consists of an orifice plate, a target plate, and side rims with effusion outlet holes, as shown in Fig. 3. The spent air flows through the outlet holes on the exit rim to the outlet plenum. The target plate is made out of Perspex (thickness $20 \mathrm{~mm}$ ). It has a low thermal conductivity and allows optical access needed for the heat transfer measurements. The target plate is observed from the outside of the outlet plenum with two CCD video cameras. Because the outflow pipe is located in the middle of the outlet plenum, which blocks the view of the camera when using one camera, two CCD video cameras are used here to catch the whole view of the target plate.

There are 81 impingement holes $(d=10 \mathrm{~mm})$ for the inline impingement plate. The ratios of jet-to-jet spacing in both directions on the impingement plate are the same $(X / d=Y / d=5)$. Because of a temperature gradient of the inlet flow from center to corner, it is necessary to install many thermocouples on the impingement plate to measure the local reference temperature that is needed for the heat transfer evaluation. In the present study, the jet temperature is used as the reference temperature to evaluate the heat transfer coefficients. Figure 4 shows the inline impingement pattern used in the scope of the present work and the positions of the thermocouples measuring the reference temperature used for the data evaluation. Because of the symmetry of the impingement plate, only half of it is presented. These thermocouples are placed directly in the center of the impinging hole at the jet exits. Three ratios of the jet-to-plate spacing $(H / d=1,2,3,4$, 
Table 1 Summary of relevant previous studies

\begin{tabular}{|c|c|c|c|c|}
\hline$(X / d, Y / d)$ & Cross flow & $H / d$ & $\operatorname{Re}$ & Author(s) \\
\hline$(1.9,1.9)$ & Minimum & $0.4,0.73,1.47,4.5$ & $1000-3800$ & Andrews et al. [4] \\
\hline$(3,3)$ & Minimum & $0.5,2,4$ & $5000-20,000$ & Garimella and Schroeder [8] \\
\hline$(3,3)$ & Maximum & $1.25,2,2.75,4,5.5$ & $14,858-58,063$ & Bailey and Bunker [10] \\
\hline $\begin{array}{l}(3,3.75) \\
(4.8,6)\end{array}$ & Maximum & $1.875,3$ & $19,552-28,870$ & Son et al. [15] \\
\hline$(4,4)$ & Minimum & $0.5,1,2,4$ & $5000-20,000$ & Garimella and Schroeder [8] \\
\hline$(4,4)$ & Minimum & $3,4,6,8,10$ & $5000-20,000$ & Geers et al. [14] \\
\hline$(4.7,4.7)$ & Minimum & $1,1.83,3.67,4.5$ & $1000-8000$ & Andrews et al. [4] \\
\hline \multicolumn{5}{|l|}{$(5,2)$} \\
\hline \multicolumn{5}{|l|}{$(5,6)$} \\
\hline$(5,4),(5,8)$ & $\begin{array}{l}\text { Maximum } \\
\text { Minimum }\end{array}$ & $1,2,3,6$ & 10,000 & Metzger et al. [3] \\
\hline$(5.6,4)$ & $\begin{array}{l}\text { Medium } \\
\text { Maximum }\end{array}$ & $2,4,6,8,12,16$ & $1300-5500$ & Obot and Trabold [5] \\
\hline$(6,6)$ & Maximum & $1.25,2.75$ & $29,520-57,522$ & Bailey and Bunker [10] \\
\hline$(6.36,6.36)$ & Maximum & $1,2,3,4$ & $5600-11,500$ & Dano et al. [12] \\
\hline$(6.8,6.8)$ & Minimum & $1.42,2.58,4.5,5.15$ & $1000-15,000$ & Andrews et al. [4] \\
\hline$(8,8)$ & Maximum & 1,2 & $10,000-40,000$ & Van Treuren et al. [6] \\
\hline$(8,8)$ & Maximum & 3 & $15,000-60,000$ & Park et al. [16] \\
\hline \multicolumn{5}{|l|}{$(10,2)$} \\
\hline$(10,4)$ & Maximum & $1,2,5,10$ & $3000-20,000$ & Brevet et al. [9] \\
\hline \multicolumn{5}{|l|}{$(10,6)$} \\
\hline \multicolumn{5}{|l|}{$(10,10)$} \\
\hline $\begin{array}{l}(10,4) \\
(10,6)\end{array}$ & Maximum & $1,2,3$ & \multicolumn{2}{|c|}{$(10,4)$} \\
\hline \multicolumn{5}{|l|}{$(10,8)$} \\
\hline$(10,4)$ & Minimum & & & \\
\hline \multirow[t]{2}{*}{$(10,8)$} & Medium & $2,4,6,8,12,16$ & $1000-21,000$ & Obot and Trabold [5] \\
\hline & Maximum & & & \\
\hline$(10,10)$ & Minimum & $1,2.5,5,10$ & $3000-35,000$ & Hollworth and Berry [2] \\
\hline
\end{tabular}

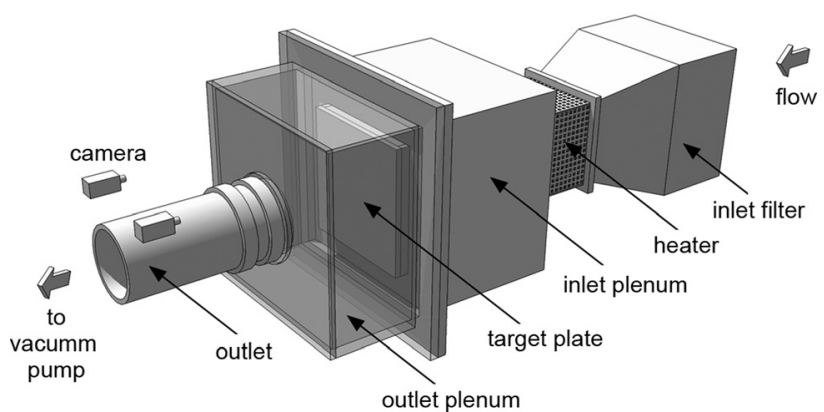

Fig. 2 Sketch of the experimental setup

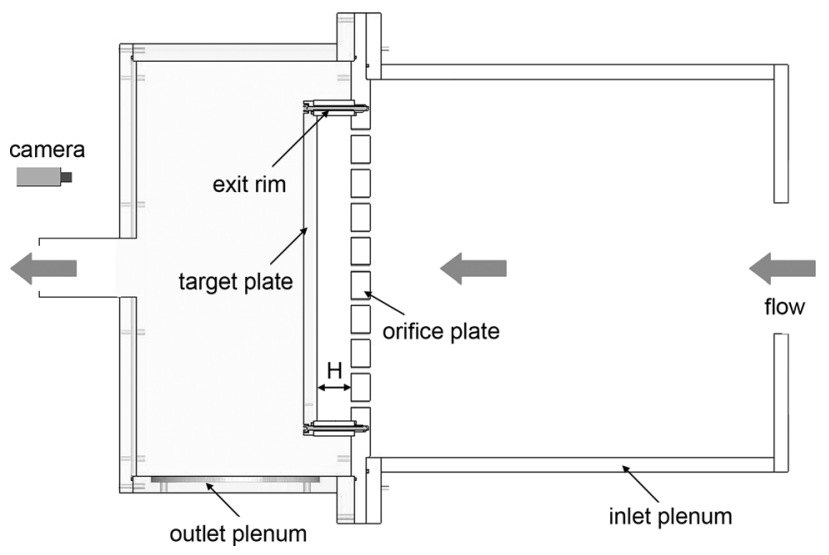

Fig. 3 The impingement model

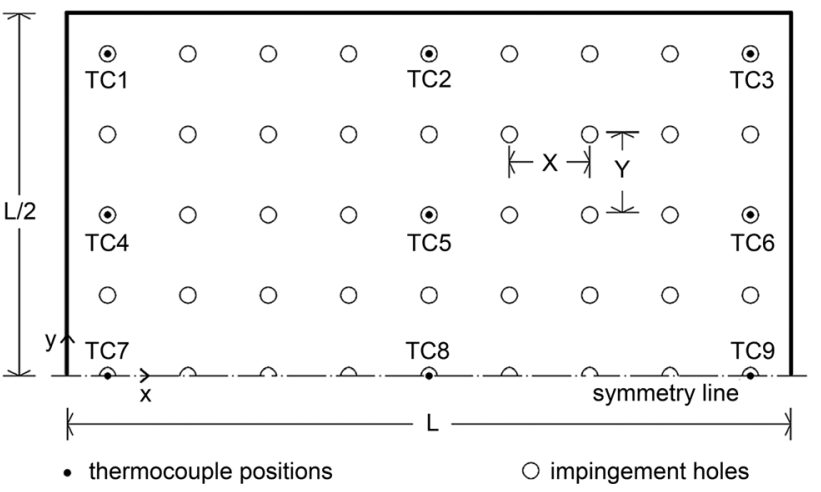

Fig. 4 The inline impingement pattern and positions of thermocouples

and 5) as well as three crossflow schemes (arranged by changing the exit rim) are investigated, as shown in Fig. 5. Three jet Reynolds numbers in the experiments are 15,000, 25,000, and 35,000. Xing et al. [17] shows that the discharge coefficients of the exit rims are nearly independent of Reynolds numbers. The discharge coefficient of $H / d=5$ is $8 \%$ lower than that of $H / d=3$. So we think the restriction of the exit rims is large enough for crossflow to be uniform.

2.2 Transient Measurement Technique. A transient method using thermochromic liquid crystals (TLC) is applied for the measurement of heat transfer, as described by Ireland and Jones [18]. Narrow bandwidth liquid crystals (with an indication temperature of $31^{\circ} \mathrm{C}$ ) are used in the present work. The TLC indication temperature is measured with a calibration unit consisting of a copper block, an electric heater, and a water cooler. The calibration unit 

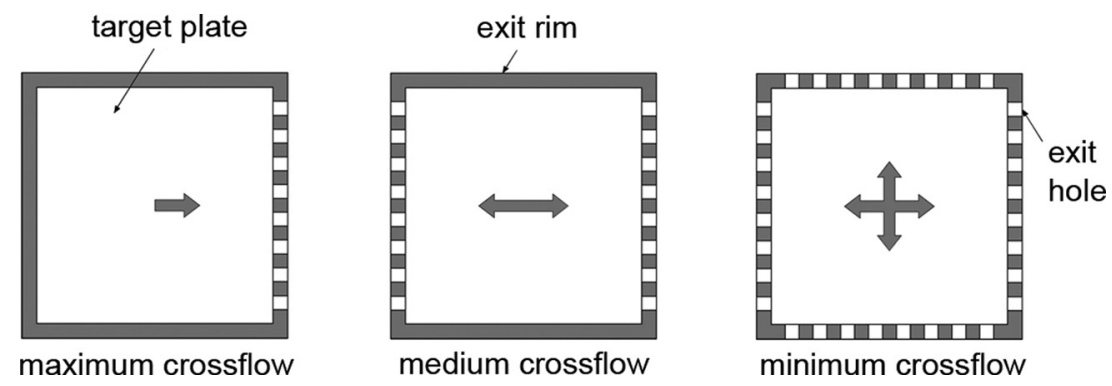

Fig. 5 The crossflow schemes

provides a $1 \mathrm{D}$ heat conduction situation, enabling easy calibration of the liquid crystal coating. The crystals are sprayed directly onto the target plate and covered with a coating of black paint in order to provide a uniform background for image acquisition.

The local heat transfer coefficients are determined using the measured wall temperature and a solution of the one-dimensional transient heat conduction equation in a semi-infinite solid. For short measurement times, one can assume a semi-infinite wall. For a flat surface, the wall surface temperature and the heat transfer coefficient are related by the expression

$$
\begin{gathered}
\Theta=\frac{T_{\mathrm{w}}-T_{0}}{T_{\mathrm{B}}-T_{0}}=1-\exp \left(\tilde{h}^{2}\right) \cdot \operatorname{erfc}(\tilde{h}) \\
\text { where } \tilde{h}=h \sqrt{\frac{t}{k \rho c}}
\end{gathered}
$$

Equation (1) has been solved numerically to obtain the heat transfer coefficient for a measured wall temperature, $T_{\mathrm{W}}$, and time, $t$, by which this temperature is reached (indicated by the color change of the liquid crystals). It is valid only for an ideal temperature step within the flow, but in reality the thermocouple records a smoother time-dependent temperature evolution. To overcome this problem, the temperature data are divided into a series of small discrete intervals $(\mathrm{N})$. Within these intervals, the temperature evolution could be considered to be an ideal temperature step. Thus, Eq. (1) can be extended for a temperature evolution according to the Duhamel principle described by Kays et al. [19],

$$
T_{\mathrm{w}}-T_{0}=\sum_{i=1}^{N} \Theta\left(t-t_{\mathrm{i}}\right)\left(T_{\mathrm{B}, \mathrm{i}}-T_{\mathrm{B}, \mathrm{i}-1}\right)
$$

where $T_{\mathrm{B}, \mathrm{i}}$ is the bulk-temperature at one specific time $t_{\mathrm{i}}$.

In a detailed impingement array, there is a spatial variation in the local temperature distribution. In the present experimental configuration, the channel widens up considerably downstream of the heater. This causes a noticeable temperature gradient from the center of the impingement plate towards the side rims. Typical temperature evolutions during an experiment are shown in Fig. 6 (for the positions of the thermocouples, refer to Fig. 4).

Due to the presence of a temperature gradient for the inlet flow, it is necessary to install a large number of thermocouples on the impingement plate to determine the local reference temperature distribution. This, in turn, was needed for precise calculation of heat transfer coefficients. Based on the temperature data for certain locations temporally, which are recorded with time by the thermocouples, a numerical method is applied to the experimental data in order to calculate the complete fluid temperature distribution during a transient experiment [20]. A two-dimensional interpolation scheme for an unknown pixel at $(x, y)$ is based on the assumption that, for each time step, the fluid temperature distribution $T(x, y, t)$ can be described by the Laplace equation. Although Laplace equation typically describes conduction within a solid, it is used as a diffusion model here for interpolation purposes only. It is solved with a finite-difference scheme for typical time

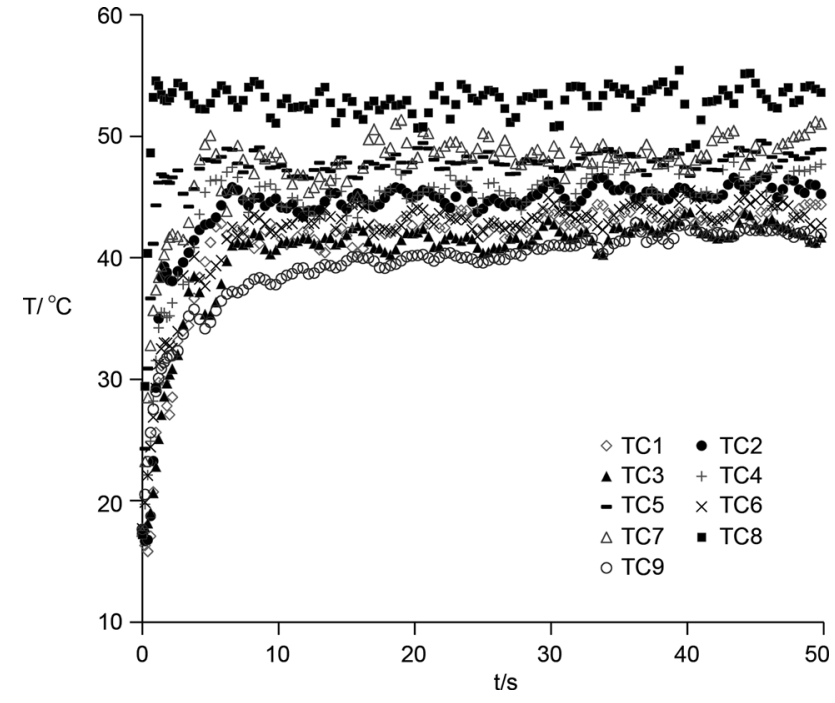

Fig. 6 Measured temperature evolution of thermocouples

steps of $0.2 \mathrm{~s}$ originating from the synchronized temperature measurements.

According to technical specifications as well as the characteristics of typical TLCs, the green color channel has been chosen to be evaluated for maximum intensity in time of a transient experiment using the data from the CCD video cameras. When the reference temperature at any pixel of the geometry has been determined, the heat transfer coefficient is determined using Eq. (3) [20].

2.3 Measurement Uncertainties. The approach used here for the measurement error analysis is based on the description by Kline and McClintock [21]. The accuracy of the measured heat transfer coefficients depended mainly on the accuracy of the thermocouples, the calibration of the liquid crystals, and the detection time.

The accuracy of the Reynolds number depends on the accuracy of the volume flow measured by the vortex meter and on the range of the static pressure sensors. The resulting uncertainty for the jet Reynolds number is below $2.5 \%$. For narrow band TLC, the transitional temperature range is $1{ }^{\circ} \mathrm{C}$ and the typical uncertainty in measuring this temperature is approximately $0.1^{\circ} \mathrm{C}$ according to Yan and Owen [22]. Temperatures $\mathrm{T}_{\mathrm{B}}$ and $\mathrm{T}_{0}$ are measured with thermocouples. A thermocouple calibration procedure shows that the error on temperature measurement is below $0.2^{\circ} \mathrm{C}$.

It should be noted that the measurement uncertainties vary with the adiabatic wall temperature and therefore are different at every position at the target plate. In particular, in the stagnation point under the impinging jets, lateral heat conduction will have an effect. According to the method by Kingsley-Rowe et al. [23], the maximum error of the Nusselt number due to lateral heat conduction has been calculated to be below $2 \%$ when the dimensionless 
temperature ratio $\Theta$, as in Eq. (1), is in the range of $0.3 \leq \Theta \leq 0.7$.

The total measurement uncertainty for the Nusselt number is below $9 \%$ for all experiments that have been carried out in this study.

\section{Results and Discussion}

3.1 Maximum Crossflow. Figure 7 shows the local Nusselt number distributions on the target plate for a Reynolds number of 35,000 and the maximum crossflow scheme, which the spent air exhausts only in one direction. Because of the symmetry of the plate and the crossflow, only one jet row zone (4th row) on the target plate has been shown. The positions of the impingement jets are clearly visible in the heat transfer pattern on the target plate. After the jet impinges on the target plate, the heat transfer rates are very high but decrease quickly towards the sides. The exhaust flow is cooled by the target flow and is then entrained back into the jet, particularly in the wall jet region. This entrainment will degrade the heat transfer. One can see that, as the crossflow increases, the heat transfer coefficient increases first. It is because the back pressure decreases the jet velocity, where the crossflow velocity is small. For jet-to-plate spacing of $H / d=1$, lower heat transfer coefficients at stagnation points are obtained, because the jet flow could not develop fully enough before reaching the target plate. Multiple jet impingement for the maximum crossflow scheme can be thought of as a coupled effect of jet impingement and channel flow caused by the crossflow. Heat transfer for the part of the channel flow can be enhanced because of the higher crossflow velocity with narrow jet-to-plate spacing, so the heat transfer performance in the downstream part is even better than that in the upstream part. Cases with jet-to-plate spacings of $H / d=2$ and $H / d=3$ get a more uniform heat transfer distribution in stagnation zones on the target plate, because the small jet-toplate spacings ensure that the jets reach the target plate before mixing with the crossflow. One can see that the local values in the stagnation zone are higher but decrease very fast for the spacing $H / d=2$. The jet-to-plate spacing $H / d=4$ results in lower heat transfer coefficients in the downstream region compared with jetto-plate spacing $H / d=3$. A larger jet-to-plate spacing allows a longer distance for the jet to mix with the recirculation flow before the jet reaches the target plate. The jet-to-plate spacing $H / d=5$ provides the lowest heat transfer because of the larger spacing and also the smaller crossflow velocity.

For quantification of the effect of jet-to-plate spacing, spanwise-averaged Nusselt numbers (averaged over half of the target plate) on the target plate are compared for different arrangements. The results are shown in Fig. 8. Apparently, the jet-to-plate spacings $H / d=1$ and $H / d=5$ provide lower heat transfer rates compared to the others. No big differences are apparent in the

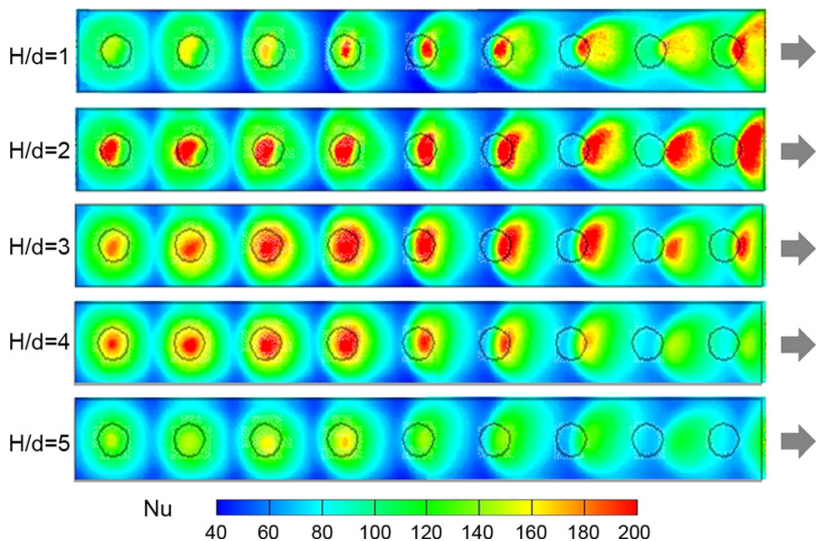

Fig. 7 Local Nusselt number distribution (maximum crossflow, $\mathrm{Re}=35,000$ )

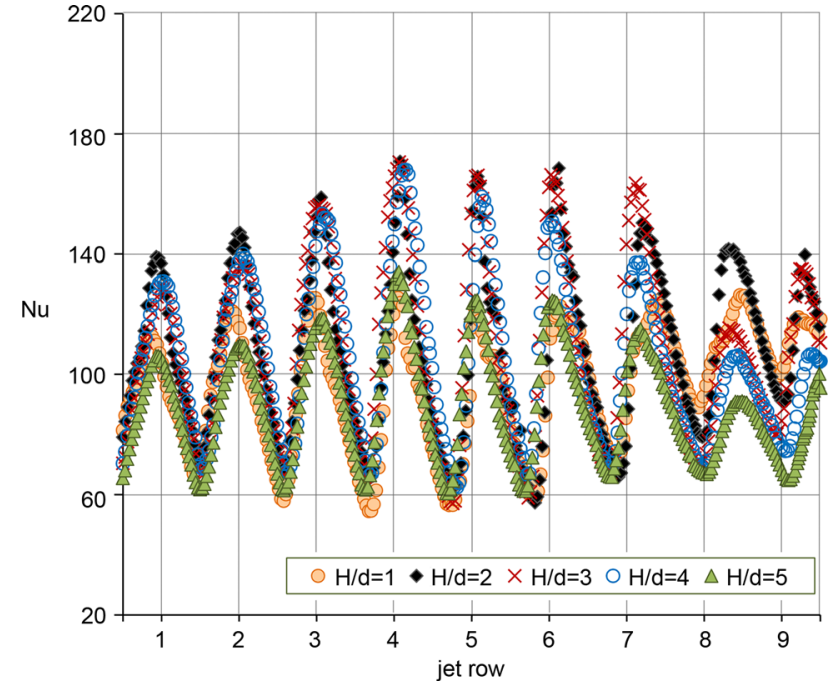

Fig. 8 Spanwise-averaged Nusselt number (maximum crossflow, $\operatorname{Re}=35,000$ )

upstream zone (jet rows from 1 to 5 ) between the jet-to-plate spacing $H / d=2,3$, and 4 . As the crossflow develops, the peak position was shifted downstream as the increased crossflow displaced the jets. The shifted distances remain nearly the same for different jetto-plate spacings. At the last two jet rows near the exit rims, lower heat transfer performance is obtained for different jet-to-plate spacing, due to the influence of the crossflow confining the jet and reducing its coverage.

The jet Reynolds number has a similar effect on impingement heat transfer. An increase in jet Reynolds number increases the local heat transfer coefficient over all of the target plate. The form of the correlation developed in Ref. [24] is

$$
\mathrm{Nu}=\phi_{1} \phi_{2} \operatorname{Re}^{\mathrm{m}} \operatorname{Pr}^{1 / 3}(\mathrm{H} / \mathrm{d})^{0.091}
$$

For the present geometry, $\mathrm{m}=0.8$. In order to normalize the heat transfer value, the area-averaged Nusselt number has been referred to $\mathrm{Re}^{0.8}$, as shown in Fig. 9. Only minor variations in overall Nusselt numbers could be observed when the jet Reynolds number is varied. This confirmed that the selected scaling is valid for the present cases. One can see that the jet-to-plate spacing $H / d=3$ results always in the highest area-averaged Nusselt number, while the spacing $H / d=5$ leads always to the lowest. The jet-to-plate spacing $H / d=1$ gets a higher normalized Nusselt number for a lower Reynolds number.

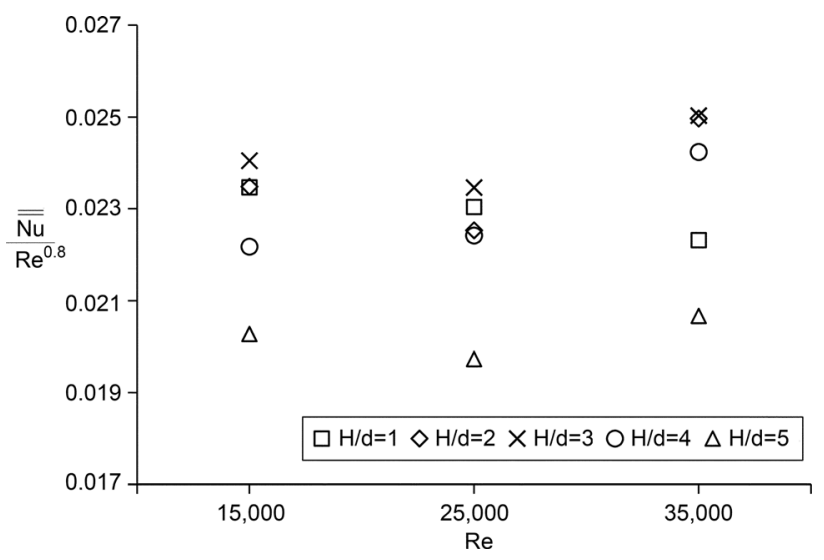

Fig. 9 Normalized area-averaged Nusselt numbers for maximum crossflow 


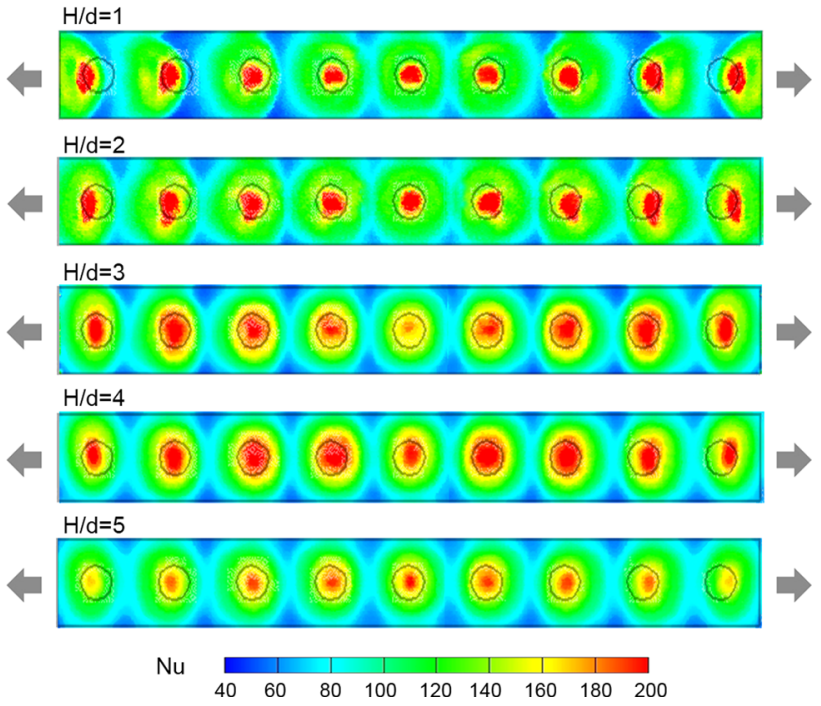

Fig. 10 Local Nusselt number distribution (medium crossflow, $\operatorname{Re}=35,000$ )

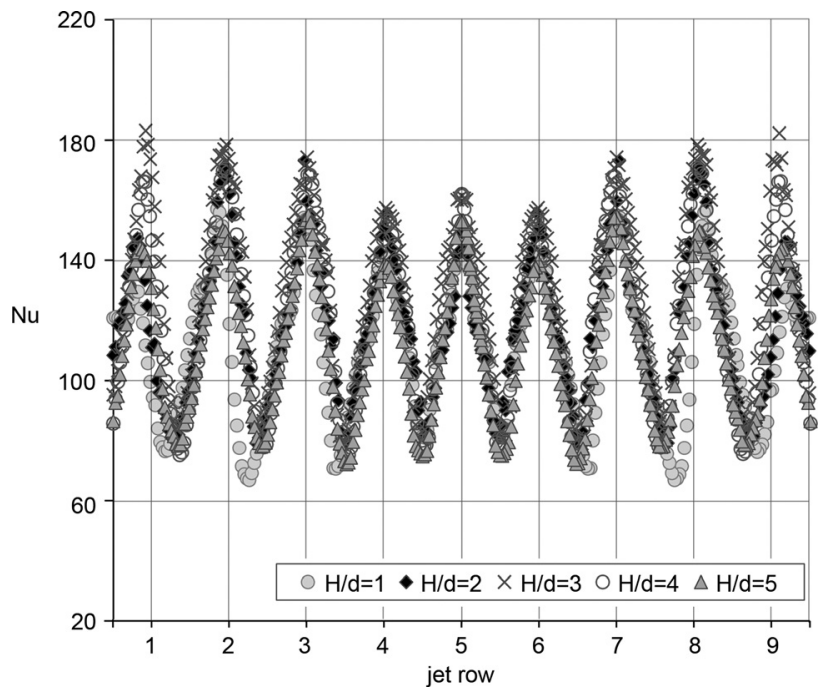

Fig. 11 Spanwise-averaged Nusselt number (medium crossflow, $R e=35,000$ )

3.2 Medium Crossflow. Figure 10 shows the local Nusselt number distributions on the target plate for a Reynolds number of 35,000 and the medium crossflow scheme, where the outlet holes are arranged in two opposite directions. For the jet-to-plate spacing $H / d=1$, lower heat transfer coefficients between the jets are visible, especially near the exit holes. This is because the jet flow could not develop sufficiently before reaching the target plate. Comparing the spacings $H / d=2$ and $H / d=3$, one can see that the local heat transfer rates in the stagnation zone are higher but decrease very fast for the spacing $H / d=2$ case. For jet-to-plate spacing $H / d=4$, the local values on the target plate are nearly the same as those for $H / d=3$. The jet-to-plate spacing $H / d=5$ results in the lowest heat transfer performance because of the larger spacing and, thus, the lower crossflow velocity.

The spanwise-averaged Nusselt numbers on the target plate are compared for different arrangements as shown in Fig. 11. No differences are apparent in the middle zone (jet rows from 4 to 6 ) for different jet-to-plate spacings. The heat transfer rates increase with an increasing crossflow.

In order to normalize the heat transfer value, the area-averaged Nusselt numbers are again scaled by $\mathrm{Re}^{0.8}$ as shown in Fig. 12. One can see that the jet-to-plate spacing of $H / d=3$ results always

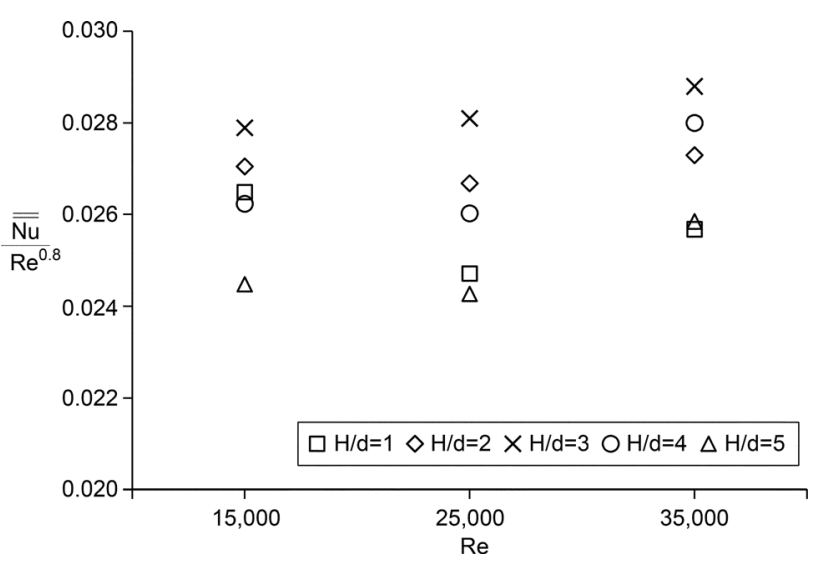

Fig. 12 Area-averaged Nusselt numbers for medium crossflow
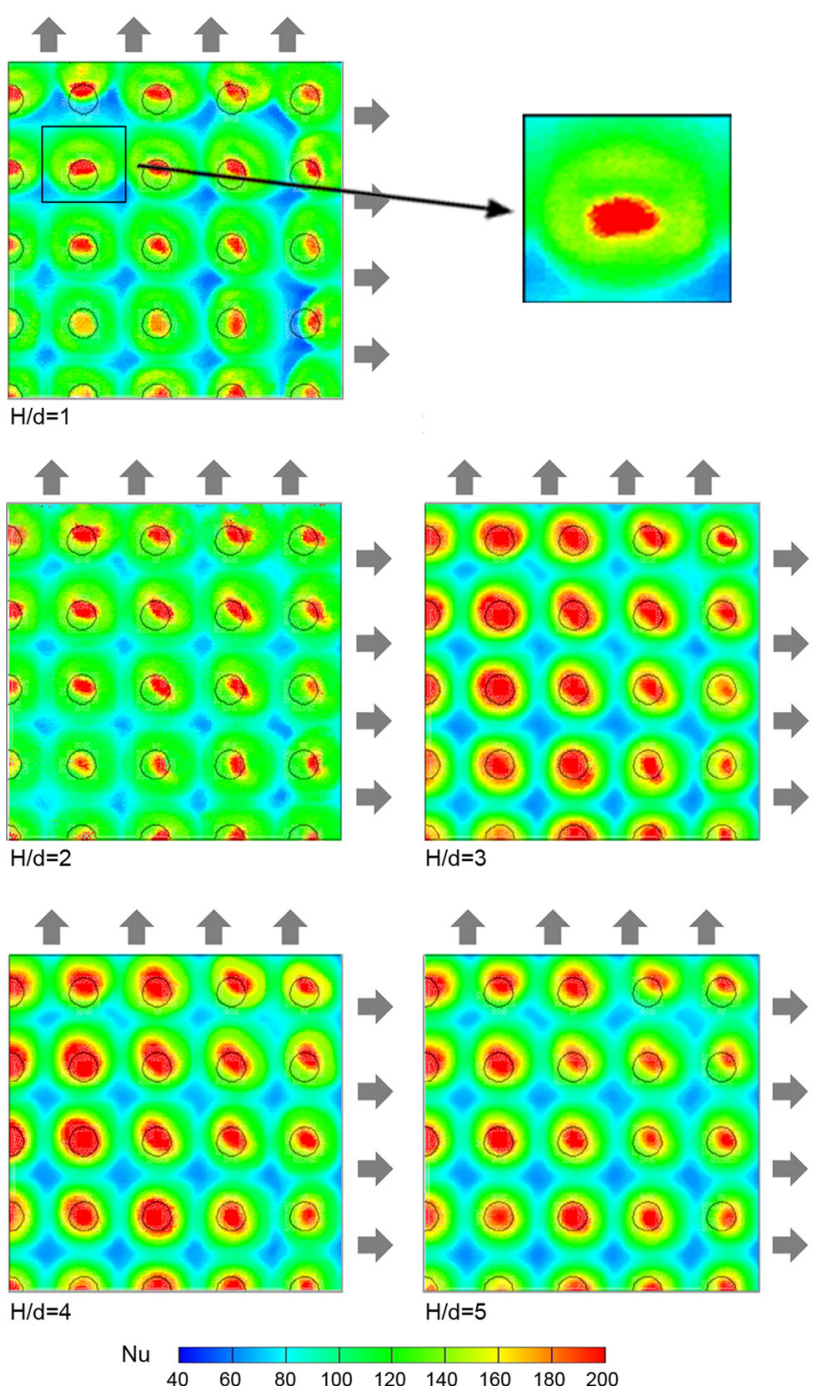

Fig. 13 Local Nusselt number distribution (minimum crossflow, $R e=35,000$ )

in the highest area-averaged Nusselt number. The jet-to-plate spacing of $H / d=1$ provides higher normalized Nusselt numbers for lower Reynolds numbers, and the spacing $H / d=5$ results in higher values for higher Reynolds numbers.

3.3 Minimum Crossflow. Figure 13 shows the local Nusselt number distributions on the target plate for a Reynolds number 


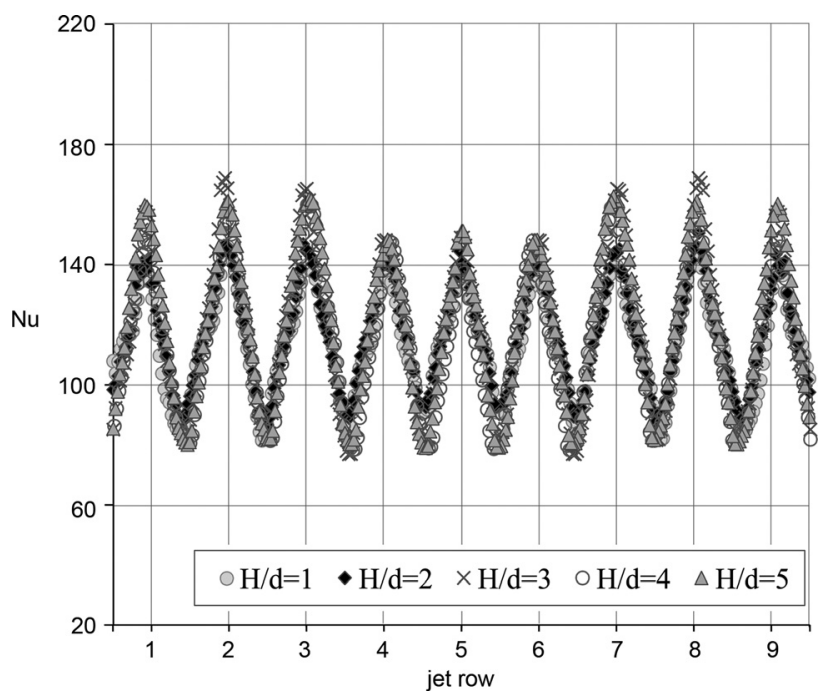

Fig. 14 Spanwise-averaged Nusselt number (minimum crossflow, $R e=35,000$ )

of 35,000 and the minimum crossflow scheme. Because of symmetry, only the upright quarter of the target plate is traced to take heat transfer measurements. One can see the apparent differences of heat transfer on the target plate with jet-to-plate spacings of $H / d=1$ to 5 . Zooming in on one jet for a jet-to-plate spacing $H / d=1$, one can see that there is a local maximum ring near the stagnation zone because the jet jumps after impinging on the plate. Comparing jet-to-plate spacings of $H / d=2$ and $H / d=3$ shows higher heat transfer values in the stagnation zone by $\mathrm{H} / \mathrm{d}=3$, while higher heat transfer values between the jets are reached by $H / d=2$. There is nearly no difference in values between cases with spacings $H / d=3$ and $H / d=4$. The jet-to-plate spacing $H / d=5$ results in lower heat transfer values in the stagnation zone compared to those for the jet-to-plate spacings $H / d=3$ and $H / d=4$.

The spanwise-averaged Nusselt numbers on the target plate are compared for different arrangements in Fig. 14. No differences are apparent for different jet-to-plate spacings. In order to normalize the heat transfer value, the area-averaged Nusselt number has again been referred to $\mathrm{Re}^{0.8}$, as shown in Fig. 15. One can see that the jet-to-plate spacing $H / d=3$ results always in the highest areaaveraged Nusselt number.

3.4 Comparisons With Literature Data. The jet-to-plate spacing $H / d=3$ is compared with literature data from Son et al. [15], Park et al. [16], and El-Gabry and Kaninski [25] and with a

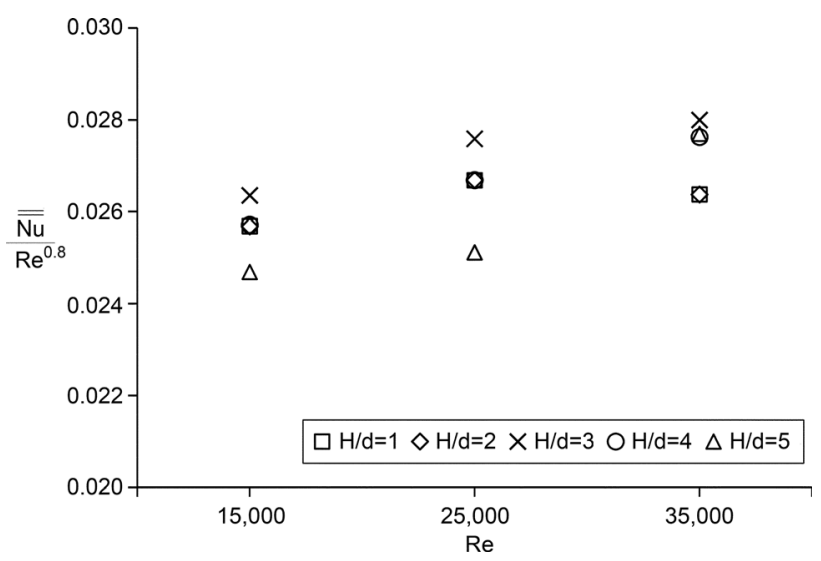

Fig. 15 Area-averaged Nusselt numbers for minimum crossflow

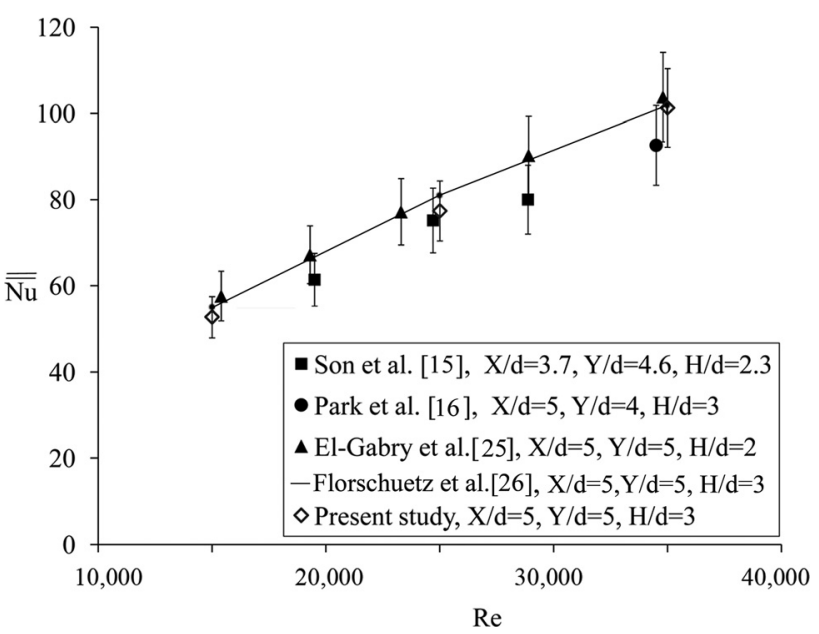

Fig. 16 Comparison with literature data for the maximum crossflow scheme (bars devote uncertainties of individual measurements)

correlation presented by Florschuetz et al. [26] for the maximum crossflow case. Figure 16 shows the comparison of the present data and the literature data. All experiments were conducted for the case of maximum crossflow. A good overall agreement is found. Although the geometric parameters for the different cases differ, the dependence of the area-averaged Nusselt number on jet Reynolds number shows generally the same trend.

\section{Conclusions}

The heat transfer characteristics in an inline impingement array with high Reynolds numbers on a flat target plate are investigated in the present work. Nusselt number distributions have been measured on the target plate using a transient liquid crystal technique. The jet exit temperature are interpolated both spatially and temporally to determine the reference temperature for the calculation of the heat transfer performance. The jet-to-plate spacing $H / d=3$ is found to be better than the others for all the crossflow schemes. The jet-to-plate spacing $H / d=1$ and $H / d=5$ provide lower heat transfer rates compared to the other spacings. The jet-to-plate spacing $H / d=2$ provides higher heat transfer rates in the stagnation zone, but the values decrease very rapidly upon traverse from that zone. The jet-to-plate spacing $H / d=4$ did not provide higher heat transfer performance with higher crossflow compared to that of $H / d=3$.

\section{Acknowledgment}

The authors kindly acknowledge the financial support of part of this work by the Friedrich- und Elisabeth Boysen Foundation.

\section{Nomenclature}

$$
\begin{aligned}
c & =\text { specific heat }(\mathrm{J} /(\mathrm{kgK})) \\
d & =\text { impingement jet diameter }(\mathrm{m}) \\
h & =\text { heat transfer coefficient }\left(\mathrm{W} /\left(\mathrm{m}^{2} \mathrm{~K}\right)\right) \\
H & =\text { jet-to-plate spacing }(\mathrm{m}) \\
k & =\text { thermal conductivity }(\mathrm{W} /(\mathrm{mK})) \\
L & =\text { target plate length }(\mathrm{m}) \\
N & =\text { discrete interval } \\
\mathrm{Nu} & =\text { Nusselt number, based on jet diameter } \\
\overline{\mathrm{Nu}} & =\text { spanwise-averaged Nusselt number } \\
\overline{\mathrm{Nu}} & =\text { area-averaged Nusselt number } \\
\mathrm{Pr} & =\text { Prandtl number } \\
\mathrm{Re} & =\text { Reynolds number, based on jet diameter } \\
T & =\text { temperature }(\mathrm{K}) \\
t & =\text { time }(\mathrm{s})
\end{aligned}
$$


$x, y=$ coordinate $(\mathrm{m})$

$X=$ spanwise jet-to-jet spacing $(\mathrm{m})$

$Y=$ streamwise jet-to-jet spacing $(\mathrm{m})$

\section{Greek Symbols}

$\begin{aligned} \rho & =\text { density }\left(\mathrm{kg} / \mathrm{m}^{3}\right) \\ \Theta & =\text { dimensionless temperature ratio }\end{aligned}$

\section{Subscripts}

$$
\begin{aligned}
0 & =\text { initial condition } \\
\mathrm{B} & =\text { bulk } \\
\mathrm{i} & =\text { index } \\
\mathrm{W} & =\text { wall }
\end{aligned}
$$

\section{References}

[1] Boyce, M. P., 2001, Gas Turbine Engineering Handbook, 2nd ed., Gulf Publishing, Woburn, MA.

[2] Hollworth, B. R., and Berry, R. D., 1978, "Heat Transfer From Arrays of Impinging Jets With Large Jet-to-Jet Spacing," ASME J. Heat Trans., 100(2), pp. 352-357.

[3] Metzger, D. E., Florschuetz, L. W., Takeuchi, D. I., Behee, R. D., and Berry, R. A., 1979, "Heat Transfer Characteristics for Inline and Staggered Arrays of Circular Jets With Crossflow of Spent Air," ASME J. Heat Trans., 101(3), pp. 526-531.

[4] Andrews, G. E., Asere, A. A., Hussain, C. I., and Mkpadi, M. C., 2005, "Full Coverage Impingement Heat Transfer: The Variation in Pitch to Diameter Ratio at a Constant Gap," AGARD, CP-390, pp. 26.1-26.12.

[5] Obot, N. T., and Trabold, T. A., 1987, "Impingement Heat Transfer Within Arrays of Circular Jets: Part 1-Effects of Minimum, Intermediate, and Complete Crossflow for Small and Large Spacings," ASME J. Heat Trans., 109(4), pp. 872-879.

[6] Van Treuren, K. W., Wang, Z., Ireland, P., Jones, T. V., and Kohler, S. T. 1996, "Comparison and Prediction of Local and Average Heat Transfer Coefficients Under an Array of Inline and Staggered Impinging Jet," ASME Paper No. 96-GT-163.

[7] San, J. Y., and Lai, M. D., 2001, "Optimum Jet-to-Jet Spacing of Heat Transfer for Staggered Arrays of Impinging Air Jets," Int. J. Heat Mass Transfer, 44, pp. $3997-4007$.

[8] Garimella, S. V., and Schroeder, V. P., 2001, "Local Heat Transfer Distributions in Confined Multiple Air Jet Impingement," ASME J. Electron. Packaging, 123(3), pp. 165-172
[9] Brevet, P., Dejeu, C., Dorignac, E., Jolly, M., and Vullierme, J. J., 2002, "Heat Transfer to a Row of Impinging Jets in Consideration of Optimization," Int. J. Heat Mass Transfer, 45, pp. 4191-4200.

[10] Bailey, J. C., and Bunker, R. S., 2002, "Local Heat Transfer and Flow Distributions for Impinging Jet Arrays of Dense and Sparse Extent," ASME, Paper No. GT2002-30473.

[11] Gao, L., 2003, "Effect of Jet Hole Arrays Arrangement on Impingement Heat Transfer," M.Sc. thesis, Louisiana State University, Baton Rouge, LA.

[12] Dano, B. P. E., Liburdy, J. A., and Kanokjaruvijit, K., 2005, "Flow Characteristics and Heat Transfer Performances of a Semi-Confined Impinging Array of Jets: Effect of Nozzle Geometry,” Int. J. Heat Mass Transfer, 48, pp. 691-701.

[13] Katti, V., and Prabhu, S. V., 2008, "Influence of Spanwise Pitch on Local Heat Transfer Distribution for In-Line Arrays of Circular Jets With Spent Air Flow in Two Opposite Directions," Exp. Therm. Fluid Sci., 33, pp. 84-95.

[14] Geers, L. F. G., Tummers, M. J., Bueniinck, T. J., and Hanjalic, K., 2008, "Heat Transfer Correlation for Hexagonal and In-Line Arrays of Impinging Jets," Int. J. Heat Mass Transfer, 51, pp. 5389-5399.

[15] Son, C., Gillespie, D., and Ireland, P., 2000, "Heat Transfer and Flow Characteristics of an Engine Representative Impingement Cooling System," ASME Paper No. 2000-GT-219.

[16] Park, J., Goodro, M., Ligrani, P., Fox, M., and Moon, H. K., 2007, "Separate Effects of Mach Number and Reynolds Number on Jet Array Impingement Heat Transfer," ASME J. Turbomach., 129(2), pp. 269-280.

[17] Xing, Y., Spring, S., and Weigand, B., 2010, "Experimental and Numerical Investigation of Heat Transfer Characteristics of Inline and Staggered Arrays of Impinging Jets," ASME J. Heat Trans., 132(9), p. 092201.

[18] Ireland, P. T., and Jones, T. V., 2000, "Liquid Crystal Measurements of Heat Transfer and Surface Shear Stress," Meas. Sci. Technol., 11, pp. 969-986.

[19] Kays, W. M., Crawford, M. E., and Weigand, B., 2004, Convective Heat and Mass Transfer, McGraw-Hill International Editions, New York.

[20] Poser, R., von Wolfersdorf, J., and Lutum, E., 2007, “Advanced Evaluation of Transient Heat Transfer Experiments Using Thermochromic Liquid Crystals," Proc. Inst. Mech. Eng., Part A, 221(6), pp. 793-801.

[21] Kline, S. J., and McClintock, F. A., 1953, "Describing Uncertainties in SingleSample Experiments," J. Mech. Eng., 75, pp. 3-8.

[22] Yan, Y., and Owen, J. M., 2002, "Uncertainties in Transient Heat Transfer Measurements With Liquid Crystal," Int. J. Heat Fluid Flow, 23, pp. 29-35.

[23] Kingsley-Rowe, J. R., Lock, G. D., and Owen, J. M., 2005, "Transient Heat Transfer Measurements Using Thermochromic Liquid Crystal: Lateral-Conduction Error,” Int. J. Heat Fluid Flow, 26, pp. 256-263.

[24] Kercher, D. M., and Tabakoff, W., 1970, "Heat Transfer by a Square Array of Round Air Jets Impinging Perpendicular to a Flat Surface Including the Effect of Spent Air," ASME J. Eng. Power, 92(1), pp. 73-82.

[25] El-Gabry, L. A., and Kaminski, D. A., 2005, "Experimental Investigation of Local Heat Transfer Distribution on Smooth and Roughened Surfaces Under an Array of Angled Impinging Jets," ASME J. Heat Trans., 127(3), pp. 532-544.

[26] Florschuetz, L. W., Truman, C. R., and Metzger, D. W., 1981, "Streamwise Flow and Heat Transfer Distributions for Jet Array Impingement With Crossflow," ASME J. Heat Trans., 103(2), pp. 337-342. 